Geoscience and Remote Sensing Symposium (IGARSS), 2016 IEEE International. 10-15 July 2016 Pages 3993-3996

http://dx.doi.org/10.1109/IGARSS.2016.7730038

http://archimer.ifremer.fr/doc/00356/46713/

(c) IEEE 2016

\title{
Ocean doppler anomaly and ocean surface current from Sentinel 1 tops mode
}

\author{
Johnsen Harald ${ }^{1,{ }^{*}}$, Nilsen Vegard ${ }^{1}$, Engen Geir ${ }^{1}$, Mouche Alexis ${ }^{2}$, Collard Fabrice ${ }^{3}$
}

${ }_{1}^{1}$ Northern Research Institute, Box 6434, N-9294 Tromsoe, Norway

2 Laboratoire d'Ocanographie Spatiale - Ifremer, 29280 Plouzane, France

${ }^{3}$ OceanDataLab, 29280 Locmaria Plouzane, France

* Corresponding author : Harald Johnsen, email address : harald.johnsen@norut.no

\begin{abstract}
:
Processing and analysis of Doppler information from Sentinel 1A Interferometric Wide (IW) and Extra Wide (EW) modes are performed for assessing the capabilities of mapping ocean surface current field. Data from Agulhas (South-Africa) and Norwegian Coast are used in combination with numerical models, higher-order satellite products, and Lagrangian drifters. Results show strong Doppler signal and dynamics from coastal areas caused by a mixture of surface current and wind/wave induced drifts at a spatial resolution of around $2 \mathrm{~km} 2$ in IW mode and $4 \mathrm{~km} 2$ in EW mode. Doppler values of up to $70 \mathrm{~Hz}$ are observed, corresponding to a surface drift velocity of $3.5 \mathrm{~m} / \mathrm{s}$. The Sentinel 1 retrieved surface current component is in reasonable agreement with the circulation models and drifter measurements. Surface current values up to $1.5 \mathrm{~m} / \mathrm{s}$ are observed in the central Agulhas current, with a standard deviation of around $0.39 \mathrm{~m} / \mathrm{s}$ with respect to Lagrangian drifters.
\end{abstract}

Keywords : Doppler effect, Sea surface, Sea measurements, Ocean temperature, Surface treatment, Antennas 
In Section 3 we first describe the procedure used for calibrating the Doppler measurements to account for instrumental and orbit effects. Secondly, the methodology for retrieving the ocean surface current (OSC) from the Doppler anomaly is outlined. In Section 4 some validation results are presented and discussed.

\section{DATA DESCRIPTION}

The following data sources are used in the analysis:

- Sentinel $1 \mathrm{WV}$, IW and EW data (earth.esa.int)

- AROME local weather model (met.no)

- ECMWF global weather model (www.ecmwf.int)

- OSCAR circulation model (oscar.noaa.gov)

- GlobCurrent data product (www.globcurrent.org)

- Lagrangian drifters (www.metocean.com)

- Sea surface temperature product (www.ifremer.fr)

- Ocean Virtual Laboratory (ovl.oceandatalab.com)

\section{INTRODUCTION}

The Doppler centroid anomaly recorded over ocean with a Synthetic Aperture Radar (SAR) can be used to obtain range directed velocity which has been demonstrated to provide valuable estimates of the near surface wind speed, ocean surface current [1],[2],[3] and sea ice sea drift [4]. The Doppler centroid (Dc) frequency recorded over ocean differs from the geometric and instrument Doppler centroids arising from antenna miss pointing, satellite orbit and attitude. This difference, called the Doppler centroid anomaly (or geophysical Doppler), is a direct measure of the line-of-sight (radial) velocity of the moving ocean surface and is thus highly sensitive to the motion of the scatterers induced by the surface currents, winds and ocean waves.

A high-precision Doppler centroid anomaly estimator was developed and implemented as part of the Sentinel-1 Level 2 ocean processor [5],[6]. The basis for our analysis is the Sentinel-1 Level 2 products from IW and EW modes collocated with Lagrangian drifters and model data. A short description of the data used is given in Section 2.
The Sentinel 1 IW and EW data are processed into a Level 2 Doppler product at a resolution of 2 and $4 \mathrm{~km}^{2}$, respectively.

\section{METHODOLOGY}

The Doppler frequency estimated from the single-look complex data consist in general of several terms:

$$
\varpi_{d c}=\varpi_{d c}^{p h y s}+\varpi_{d c}^{g e o}+\varpi_{d c}^{e l e c}+\Delta \varpi_{d c}
$$

where $\varpi_{d c}^{\text {phys }}$ is the geophysical term, $\varpi_{d c}^{\text {geo }}$ is the geometric/attitude term, $\varpi_{d c}^{\text {elec }}$ is the antenna electronic miss pointing, and $\Delta \varpi_{d c}$ is a residual error coming from imperfect prediction of the non geophysical terms or other unknown biases. In order to derive an estimate of the geophysical Doppler $\left(\varpi_{d c}^{\text {phys }}\right.$ ), a careful prediction and removal of the non-geophysical contributions from Eq.(1) are needed.

\subsection{Doppler calibration}

The geometric and the antenna electronic miss pointing terms are initially compensated for within the Sentinel 1 Level 2 processor. However, it turns out that the geometric 
Doppler computed from the attitude described by the quaternions does not predict the Dc estimated from the SAR data. A significant latitude dependency is observed in the estimated Dc, but not in the geometric Doppler (see Fig. 1).

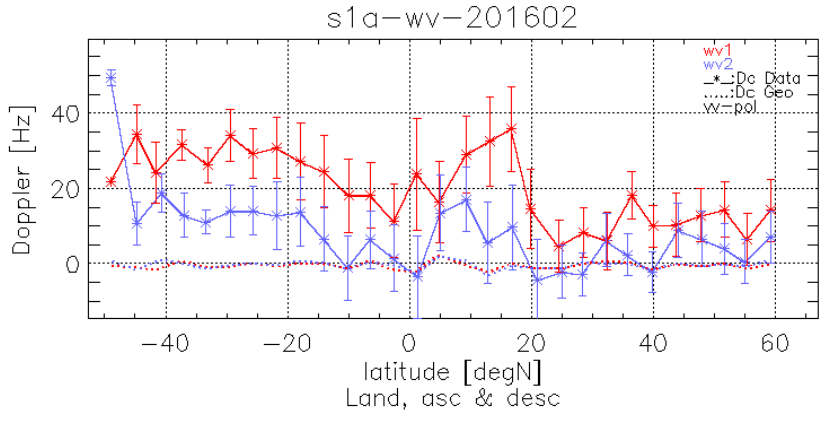

Figure 1: Latitude dependency in Dc estimated from S1A WV acquired of land areas. Data from February 2016. The error bars are the variability of the Dc estimates. The dotted lines are the corresponding geometric Doppler.

The electronic miss pointing Doppler predicted by the antenna model describes reasonably well the relative variations as function of elevation angles. However, the absolute Doppler value and the jumps in Doppler overs swaths are not always well predicted. In Figure 2 is shown the Dc estimates as function of incidence angles for the IW swaths and the corresponding electronic miss pointing Doppler computed from the antenna model. Similar results are observed for the EW mode.

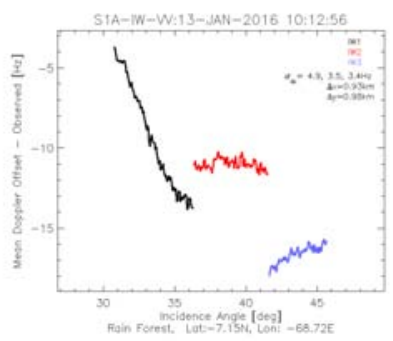

a)

Figure 2: a) Dc as function of incidence angle estimated from S1A IW mode acquired over rain forest, b) corresponding Doppler profiles estimated from antenna model.

A recalibration has been applied to the estimated Dc values to compensate at best the observed deviations shown in Figs. 1 and 2. The approach selected for our analysis is as follows:

a. Remove the electronic miss pointing Doppler from the observed Doppler, $\left(\delta \varpi \equiv \varpi_{d c}-\varpi_{d c}^{\text {elec }}\right)$ using the antenna model

b. Make the residual Doppler, $\delta \varpi$ continuous from one swath to another by merging the Doppler values in azimuth at the swath borders

c. Use land areas within the scene to absolute calibrate the residual Doppler, $\left(\varpi_{d c}^{p h y s} \equiv \delta \varpi-\langle\delta \varpi\rangle_{L A N D}\right)$. Here $\langle\delta \varpi\rangle_{L A N D}$ is the mean Doppler over land areas within the swath.
The residual Doppler is then an estimate of the Doppler anomaly or the geophysical Doppler ( $\varpi_{d c}^{p h y s}$ ) of Eq.(1).

\subsection{Doppler anomaly analysis}

The Doppler anomaly contains information of the ocean surface wind field, sea state and ocean surface current. In order to retrieve the surface current, the contributions from wind/wave induced motion must be predicted and removed. The wind/wave Doppler contribution $\left(\varpi_{d c}^{\text {cdop }}\left(\underline{U}_{10}\right)\right)$ is predicted using the CDOP [3] with input the best possible wind field, $\underline{U}_{10}$ extracted from numerical weather model. An estimate of the ocean surface current (OSC) induced Doppler is then given as:

$$
\text { (2) } \quad \varpi_{d c}^{o s c}=\varpi_{d c}^{p h y s}-\varpi_{d c}^{c d o p}
$$

Eq.(2) is then converted to ground range radial surface current by the relation:

$$
U_{r}=\frac{\varpi_{d c}^{o s c}}{2 k_{r a d} \sin \theta}
$$

where $k_{\text {rad }}$ is the radar wavenumber and $\theta$ is the local incidence angle. The $U_{r}$ values are collocated with Lagrangian drifters and circulation models for validation.

For the Norwegian Coastal areas, the wind vector field is provided from the AROME model with a spatial resolution of $2.5 \mathrm{~km}$. For the Agulhas area, the global wind model from ECMWF is used. For the Agulhas areas, the collocated Oscar current field and the GlobCurrent field are extracted using the Ocean Virtual Laboratory toolbox.

\section{RESULTS}

The analysis of Sentinel 1 Doppler anomaly shows strong signatures and dynamic of surface current and wind/wave induced drifts. In Fig. 3 is shown an example of S1A IW ascending acquisition over the Agulhas current outside South-Africa. The data are processed into a Level 2 product with a spatial resolution of around $2 \mathrm{~km}^{2}$.

A strong geophysical Doppler signal (up to $70 \mathrm{~Hz}$ ) from the Agulhas current is observed (red color) near the coastline in Fig. 3a, while in the lower part of the image a mixture of wind/wave induced Doppler signal is observed (yellow/blue colour). A ground range velocity (Fig. 3b) of up to $3.5 \mathrm{~m} / \mathrm{s}$ is observed in the central current area. A negative velocity means towards the radar look direction. A Lagrangian drifter was within the area at the time of acquisition as indicated in Fig. 3b (red arrow), with a velocity of $1.1 \mathrm{~m} / \mathrm{s}$ and direction of $228 \mathrm{degN}$. In this case the wind speed and direction is around $9 \mathrm{~m} / \mathrm{s}$ and $230 \mathrm{degN}$, contributing significantly to the observed S1A velocity. 


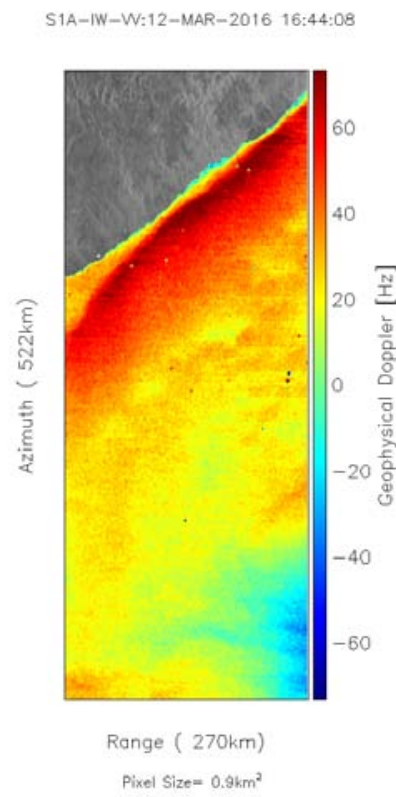

a)

Figure 3: Sentinel 1A IW Doppler anomaly (a) and the ground range velocity component (b) from ascending mode acquisition over the Agulhas area outside South-Africa. The Lagrangian drifter position and direction is indicated with a red arrow in (b). The velocity from the drifter was $1.1 \mathrm{~m} / \mathrm{s}$.

A second test area is outside the North-Norwegian coast where the continental shelf and the coastal current are close to the coastline. The area is also exposed to low-pressure systems from North-Atlantic with strong atmospheric fronts. Is it expected that the Doppler anomaly is dominated by wind/wave effects, and less by coastal current. Fig. 4 shows a Sentinel 1A IW ascending acquisition from the coast of North-Norway. The AROME model (Fig. 4c) together with CDOP is used to predict and remove the wind/wave signal from the S1A Doppler anomaly. The final ocean surface current field (ground range component) is shown in Fig. 4c. A coastal current signal component of around $0.4 \mathrm{~m} / \mathrm{s}$ is retrieved from the Doppler anomaly.

Altogether 32 scenes in IW and EW were analysed using the procedure described above. Scatterplots of the surface current speed (ground range component) from S1A versus Oscar, GlobCurrent and Lagrangian drifter, are given in Fig. 5 and 6. No filtering is applied to the data. A standard deviation of $0.39 \mathrm{~m} / \mathrm{s}$ and a bias of $0.38 \mathrm{~m} / \mathrm{s}$ are observed between S1A and Lagrangian drifters. The main contribution to the standard deviation and bias is expected to be the uncertainty in attitude as well as in the wind field used to remove the contribution to the Doppler anomaly from wind and waves.

For future work, more data will be collected and the use of S1 wind field itself will be exploited in the analysis. The foreseen improved prediction of geometric and electronic miss pointing Doppler will improve the Sentinel 1 Doppler anomaly measurements.
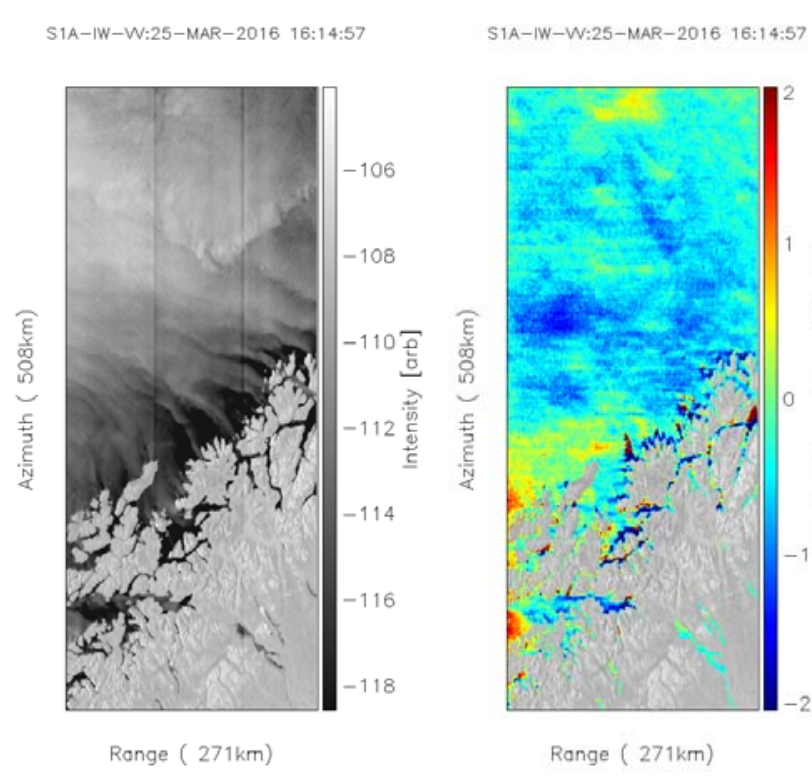

a)

b)

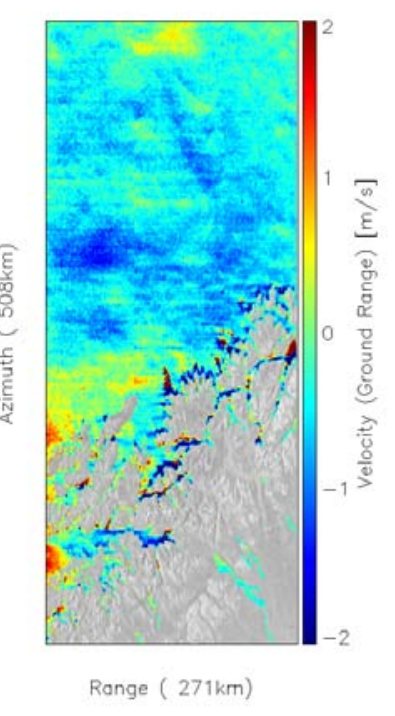

Pixel Size $=0.9 \mathrm{~km}^{2}$
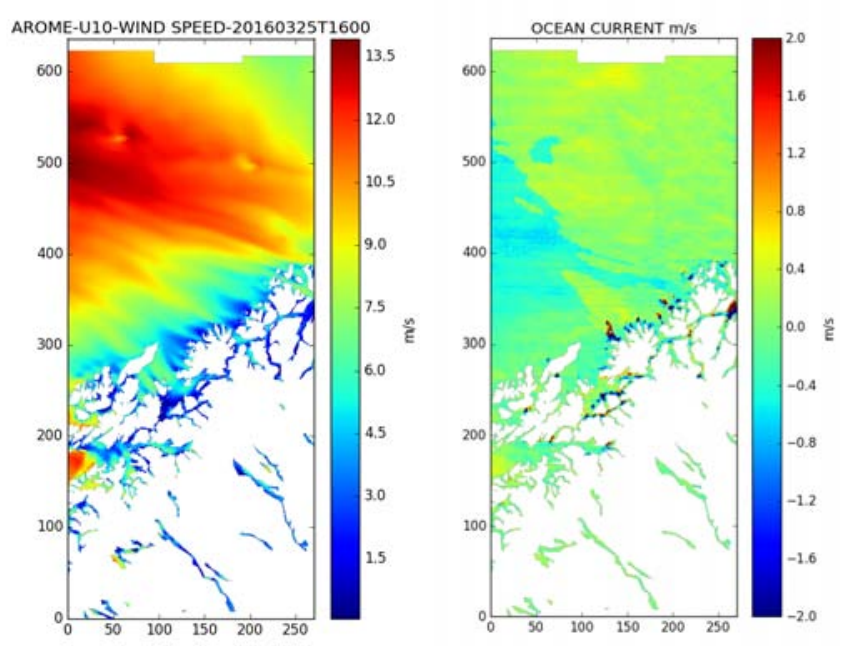

c)

d)

Figure 4: Sentinel 1A IW intensity (a), ocean surface velocity (ground range component) (b), AROME wind speed (c), and retrieved ocean surface current (ground range component) (d). 


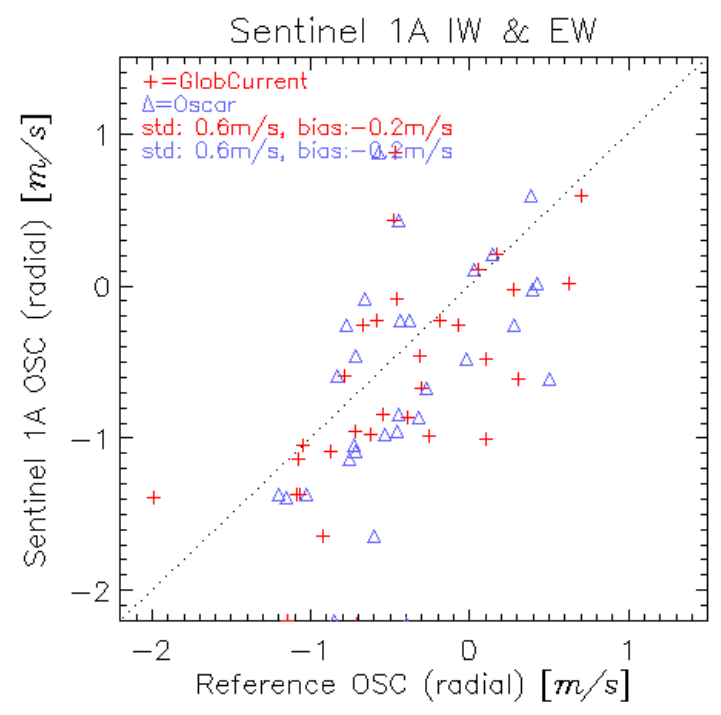

Figure 5: Scatterplot of ocean surface velocity (radial component) from S1A versus Oscar and GlobCurrent.

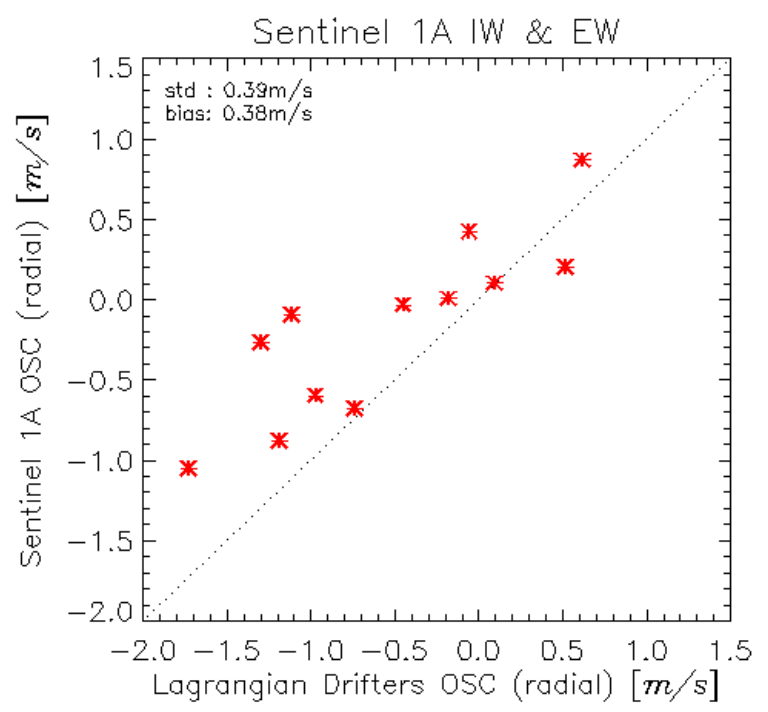

Figure 6: Scatterplot of ocean surface velocity (radial component) from S1A versus Lagrangian drifter data.

\section{Acknowledgement:}

This work is performed under the S1 Mission Performance Centre activities and the SEOM S1-4SCI Ocean Study, funded by ESA/ESRIN, and CIRFA - 237906/O30 funded by Norwegian Research Council.

\section{REFERENCES}

[1] B. Chapron, F. Collard, F. Ardhuin, "Direct measurements of ocean surface velocity from space: Interpretation and validation", J. Geophysical Research, Vol. 110, No. C7, pp. 10.1029, 2005

[2] J. A. Johannessen, B. Chapron, F. Collard, V. Kudryavtsev, A. Mouche, D. Akimov, K. F. Dagestad, "Direct ocean surface velocity measurements from space: Improved quantitative interpretation of Envisat ASAR observations", Geophysical Research Letters, Vol. 5, doi:10.1029/2008GL035709, 2008

[3] A. Mouche, F. Collard, B. Chapron, K. F. Dagestad, G. Guittion, J. A. Johannessen, V. Kerbaol, M. W. Hansen, "On the use of Doppler shift for sea surface wind retrieval from SAR", IEEE Trans. Geoscience and Remote Sensing, Vol. 50, pp.29012909, doi:10.1109/TGRS.2011.2174998, 2012

[4] T. Kræmer T., H. Johnsen, C. Brekke, "Emulating Sentinel-1 Doppler Radial Ice Drift Measurements Using Envisat ASAR Data", IEEE Trans. Geoscience and Remote Sensing, Vol.12, No.53, DOI: 10.1109/TGRS.2015.2439044, pp: 6407 - 6418, August 2015

[5] G. Engen, H. Johnsen, "Sentinel-1 Doppler and Ocean Radial Velocity Algorithm Definition”, ESA Doc. S1-TN-NRT-53-0658, ISBN 978-82-7492-311-9, ISSN 1890-5226, v1.4, 2015

[6] G. Engen, H. Johnsen, Y. Larsen, "Sentinel-1 geophysical Doppler product - performance and application", Proc. of EUSAR2014, Berlin, 3-5 June 2014 